\title{
ENCEINTE ÉTANCHE DE LA CENTRALE ÉLECTRIQUE DE BLAYE : ESSAIS ET MESURES SUR LES TIRANTS D'ANCRAGE
}

\author{
Ground anchors : tests and measurements
}

\author{
par \\ G.-Y. FENOUX \\ Directeur des Etudes de Solétanche - Paris - France
}

\section{SOMMAIRE}

Le projet de fondation de l'usine E.D.F.. sur radier semi-enterré, comporte la substitution par du sable compacté à sec des couches de vase en place (1 million de mètres cubes à évacuer).

Pour ce faire, une enceinte étanche en paroi moulée a été retenue. Dégagée sur $17.5 \mathrm{~m}$ de hauteur. supportant outre la poussée des terres sur cette hauteur celle de l'eau sur $15 \mathrm{~m}$, la paroi a $30 \mathrm{~m}$ de hauteur. Elle est ancrée en pied dans le substratum (graviers et marnocalcaire) et tenue en tête par des tirants.

De $200 \mathrm{t}$ de force de service, de $\mathbf{5 5} \mathrm{m}$ de longueur totale (dont $25 \mathrm{~m}$ d'ancrage), au nombre de 832, les tirants ont fait l'objet d'essais et de mesures, tant préalables qu'au cours du chantier.

Testés à 1.2 la charge de service, les tirants ont été bloqués à $\mathbf{0 . 5}$ fois celle-ci $(100 \mathrm{t})$, afin de limiter le mouvement en arrière de la paroi. Par la suite, on attendait un mouvement en avant (vers la fouille) de la paroi en cours de terrassement avec mise en charge complémentaire progressive des tirants.

Les mouvements de la paroi et les efforts dans les tirants ont été suivis avec attention et comparés avec les estimations préalables.

\section{SUMMARY}

The foundation project for the E.D.F. power station is a semi-buried raft, involving the replacement of the in situ soft clays and muds by a sand compacted in the dry. (1 million $\mathrm{m}^{3}$ of soil to be removed).

To achieve this, an impervious perimetral diaphragm wall was adopted. The wall was $30 \mathrm{~m}$ deep. and was uncovered over $17.5 \mathrm{~m}$, retaining both the soil and $15 \mathrm{~m}$ of water behind. The wall is toed into gravels and marly limestone and is held at the top by ground anchors.

The $\mathbf{8 3 2}$ ground anchors have a working load of $200 \mathrm{t}$, total length $\mathbf{5 5} \mathrm{m}$, and were the object of tests and measurements as much before as after the earthmoving.

Tested to 1.2 times their working load, the ground anchors were blocked at $\mathbf{0 . 5}$ this value (i.e. at $100 \mathrm{t}$ ) in order to limit the backward movement of the wall. As the earthmoving proceeded it was expected that the wall would move towards the excavation, gradually increasing the load on the anchors.

The wall movements and the loads in the ground anchors were carefully monitored and were compared with the estimations made beforehand.

\section{EXPOSE DU PROBLEME}

Le site du Blayais, situé en bordure rive droite de la Gironde, à environ $50 \mathrm{~km}$ au nord-ouest de Bordeaux, a été retenu par l'EDF pour la construction d'une centrale électrique nucléaire.

Il s'agit d'une zone marécageuse, avec la coupe schématique suivante :

$$
\begin{aligned}
& \mathrm{de}+2.5 \text { NGF à }-13.0: \text { vase }(\varphi=0, c=0.4 \text { à } \\
& \left.4 \mathrm{t} / \mathrm{m}^{2}\right) \text {; } \\
& \text { de }-13.0 \text { à }-19.0 \text { : alluvions sablo-graveleu- } \\
& \text { ses (graves) }\left(\varphi=35^{\circ}\right) \text {; } \\
& \text { de }-19.0 \text { à }-26.0 \text { : marno-calcaire de l'éo- } \\
& \left(\varphi=0, c=15 \mathrm{t} / \mathrm{m}^{2}\right) \text {; }
\end{aligned}
$$

en-dessous de $\quad-26.0$ : sable de l'éocène.

On rencontre deux nappes: une nappe de surface, qui varie de $1 \mathrm{~m}$ environ avec la marée au voisinage immédiat du terrain naturel; une nappe profonde baignant l'éocène avec un niveau statique voisin de +1.50 N.G.F. Bien qu'hétérogène, le marnocalcaire est, en grand, peu perméable.
Afin de mettre le site hors d'eau et rendre possible la circulation, le terrain naturel a été partiellement remblayé à la cote +4.50 , laquelle correspond sensiblement à celle de la digue de protection des crues de la Gironde.

Les ouvrages à fonder, sur radier enterré, sont calés à des niveaux variant entre -13 N.G.F. (station de pompage), -7.50 (bâtiment combustible) et -2.50 (groupe et salle des machines).

Le parti de fondation adopté : radier, implique une substitution complète des couches de vase, toute idée de consolidation ne résistant pas à un examen même superficiel. Il s'agit donc de réaliser une enceinte descendue au toit des graves $(17.50 \mathrm{~m}$ de hauteur totale de +4.50 à -13 ), dégageant un rectangle de $210 \times 265 \mathrm{~m}$ pour 2 groupes de $900 \mathrm{MW}$, étanche, stable latéralement et vis-à-vis des sous-pressions, qui permette d'ériger à sec la station de pompage et, sur remblai compacté de 6 à $10 \mathrm{~m}$ d'épaisseur, les bâtiments réacteur, combustible, massif de groupe, salle des machines. 


\section{CHOIX D'UNE SOLUTION}

La solution de base consiste à faire « comme d'habitude ", lorsqu'il y a de la place, une fouille talutée avec un écran étanche périphérique (solution talutée) (fig. 1).

L'écran étanche, en paroi, a $28 \mathrm{~m}$ de hauteur totale. Le terrassement des vases est réalisé sous l'eau pour éviter tout glissement des talus à $2 / 1$. Une recharge en sable, également réalisée sous l'eau, fait passer la pente des talus de 2.1 à 3.1. Une couche de tout-venant, déposée à sec, assure une protection contre l'érosion des intempéries. Une telle solution représente 40 à $45000 \mathrm{~m}^{2}$ d'écran, $1600000 \mathrm{~m}^{3}$ de terrassement, $300000 \mathrm{~m}^{3}$ de remblai sous l'eau,

La solution type paroi verticale, dont plusieurs formes sont envisageables, supprime $600000 \mathrm{~m}^{3}$ d'enlèvement de vase et de remblaiement. Elle écarte toute impasse sur la stabilité des talus, limite le risque d'anomalie d'étanchéité naturelle du fond marnocalcaire (16.5 ha dans la solution de base, 6 , seulement, dans la variante), et rapproche de $80 \mathrm{~m}$ la station de pompage de la Gironde.

La paroi verticale "classique » se dessine plane avec des tirants forés précontraints (fig. 2.). Les efforts étant considérables $(17.5 \mathrm{~m}$ de poussée des terres et 15.5 de poussée d'eau : un véritable barrage de basse chute), quatre lignes de tirants sont nécessaires. Dès lors, le terrassement est à faire à sec, en ménageant au fur et à mesure les plateformes utiles pour forer les tirants. Cette solution se caractérise de ce fait par des délais voisins du double des délais normaux de la solution de base.

D'où le choix de la solution retenue, avec terrassement par dragage sous l'eau (le terrassement sous l'eau a priori économique équilibrant une paroi à contreforts plus lourde qu'une paroi plane) qui permet de respecter les délais impartis.

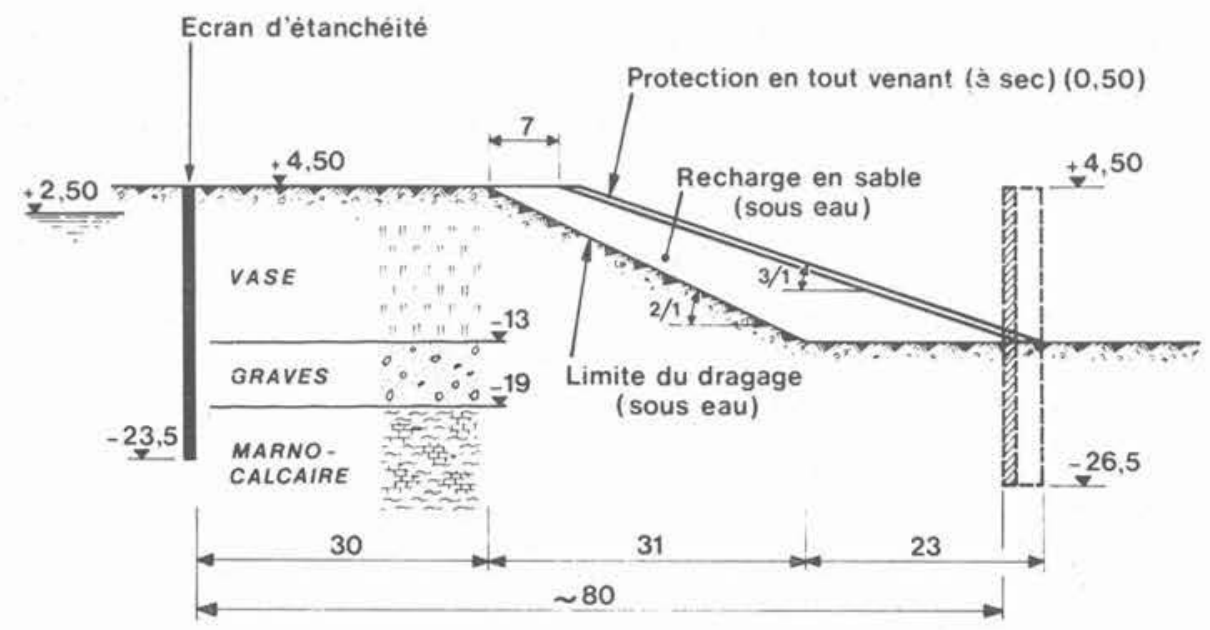

Fig, 1. - Coupe type de la solution de base.

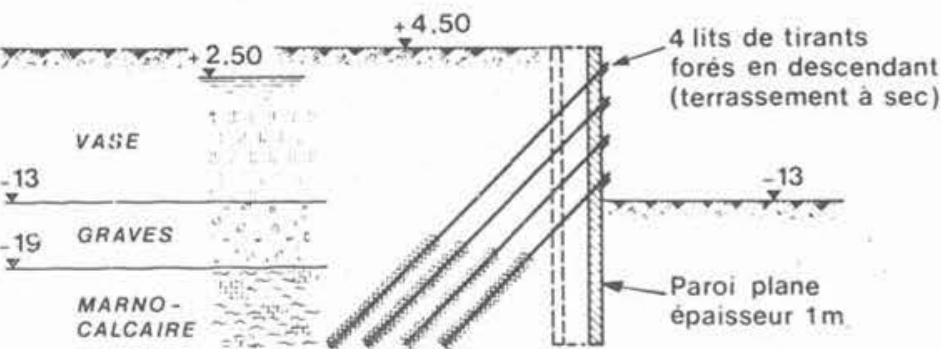

DESCRIPTION DE LA SOLUTION

En plan, la paroi s'inscrit dans un rectangle de $275 \times 219 \mathrm{~m}$ (fig. 3 ) en bordure immédiate de la digue de protection de la Gironde.

Le schéma d'exécution (fig. 4) que les délais imposent implique que le soutènement soit stable dès le début de la phase 2, c'est-à-dire le dragage. La paroi équilibre les efforts de poussée en s'appuyant en tête sur des tirants et en pied sur le terrain en place. Le moment maximum atteint de ce fait plus de $1000 \mathrm{tm}$ par mètre linéaire, la paroi est du type à « contrefort» (fig. 5).

Chaque panneau (plot, ou contrefort) a $4.70 \mathrm{~m}$ de développement et doit, pour être équilibré, recevoir en tête une réaction horizontale de $5900 \mathrm{KN}$. Cette réaction est assurée par quatre tirants inclinés en moyenne à $40^{\circ}$ sur l'horizontale et de $1930 \mathrm{KN}$ de tension unitaire en service (fig. 6).

L'équilibre général et la disposition des couches conduisent à prévoir des tirants d'une longueur totale de $55 \mathrm{~m}$ dont $30 \mathrm{~m}$ de longueur libre et $25 \mathrm{~m}$ de scellement.

La paroi a une hauteur totale moyenne de $31 \mathrm{~m}$, la longueur de fiche $(13.5 \mathrm{~m})$ étant intermédiaire entre les résultats des calculs en "butée simple » et en « semiencastrement». L'épaisseur normale de $0.80 \mathrm{~m}$ est portée à $1 \mathrm{~m}$ dans les contreforts pour des raisons essentiellement pratiques : passage des tirants en tête, disposition des armatures dans les zones de recouvrement. 


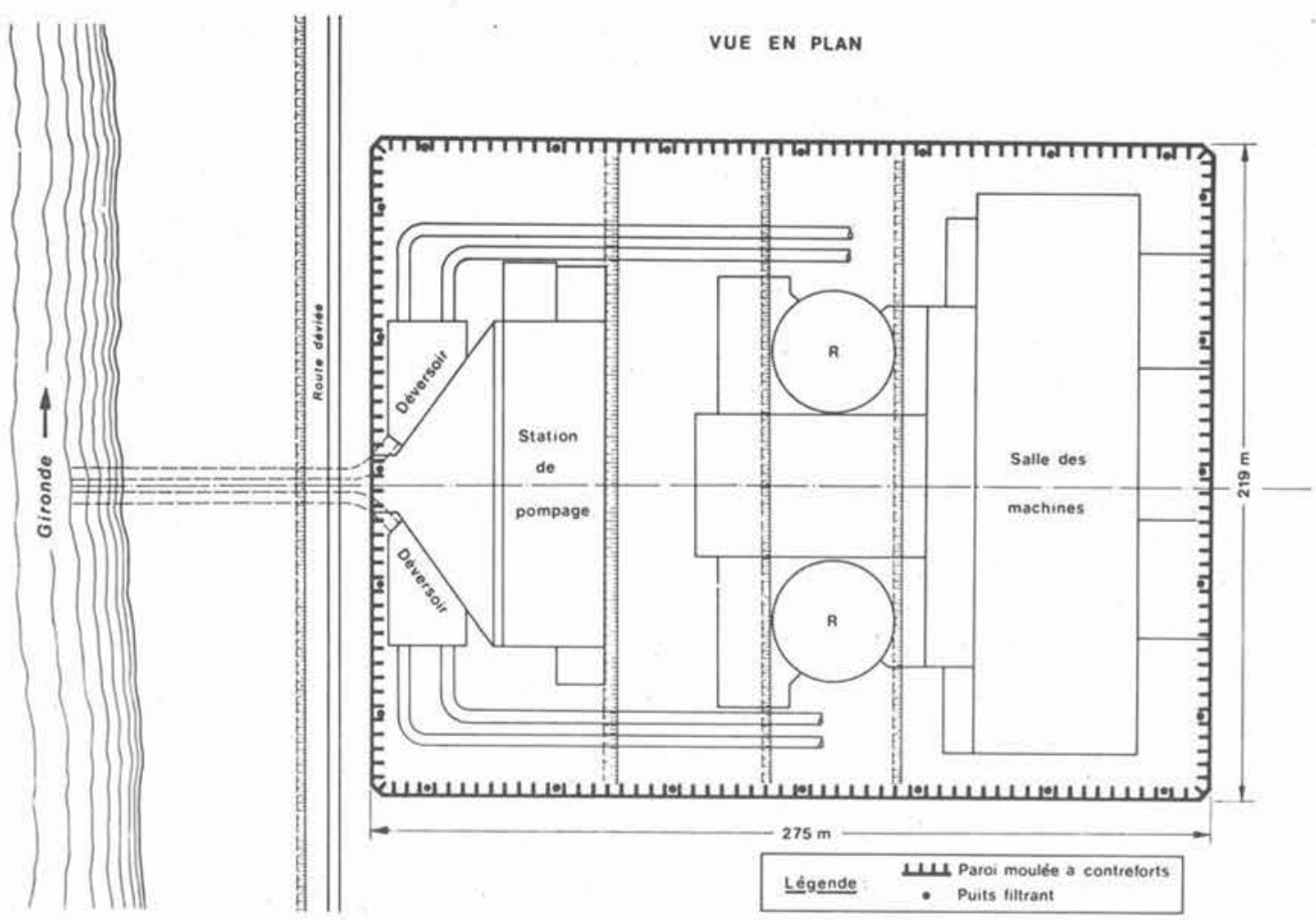

Fig. 3. - Vue en plan de l'enceinte.

(1) Exécution de la paroi et des tirants

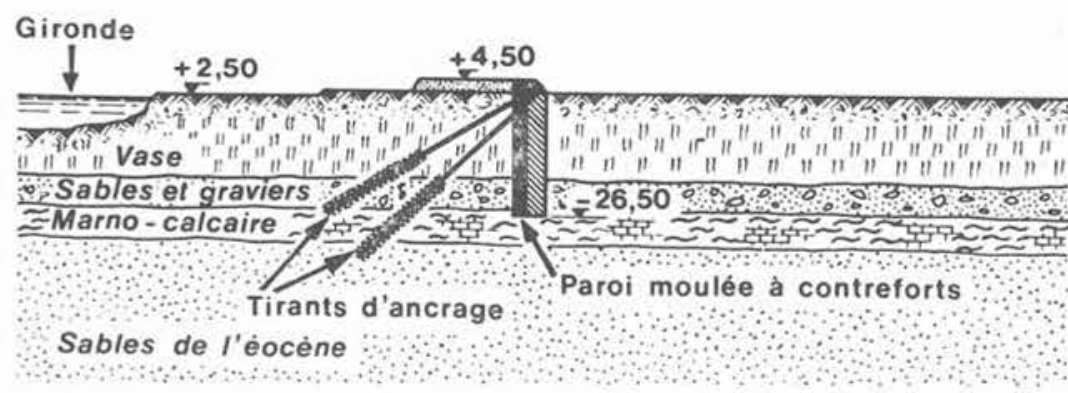

Fig. 4. - Schéma d'exécution.

(2) Terrassement de la fouille par dragage

(3) Pompage de l'eau
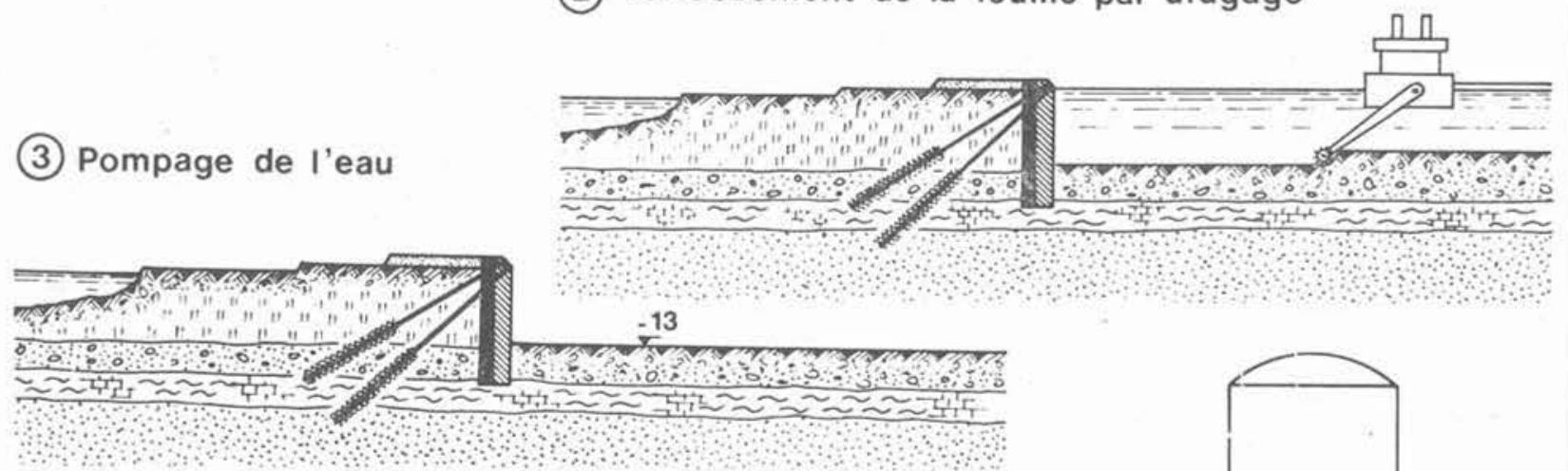

(4) Construction génie civil

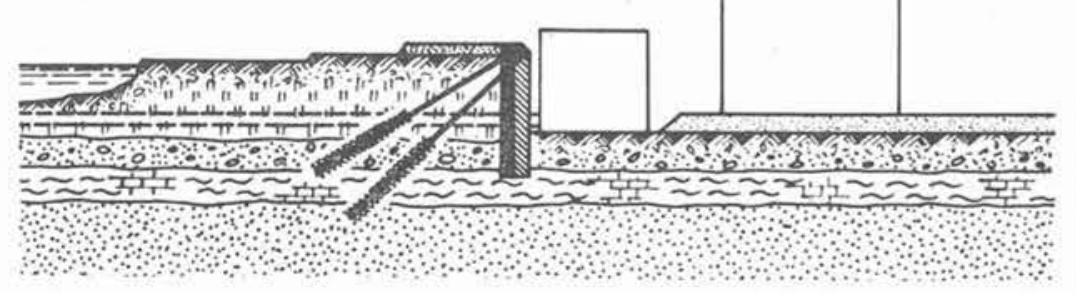



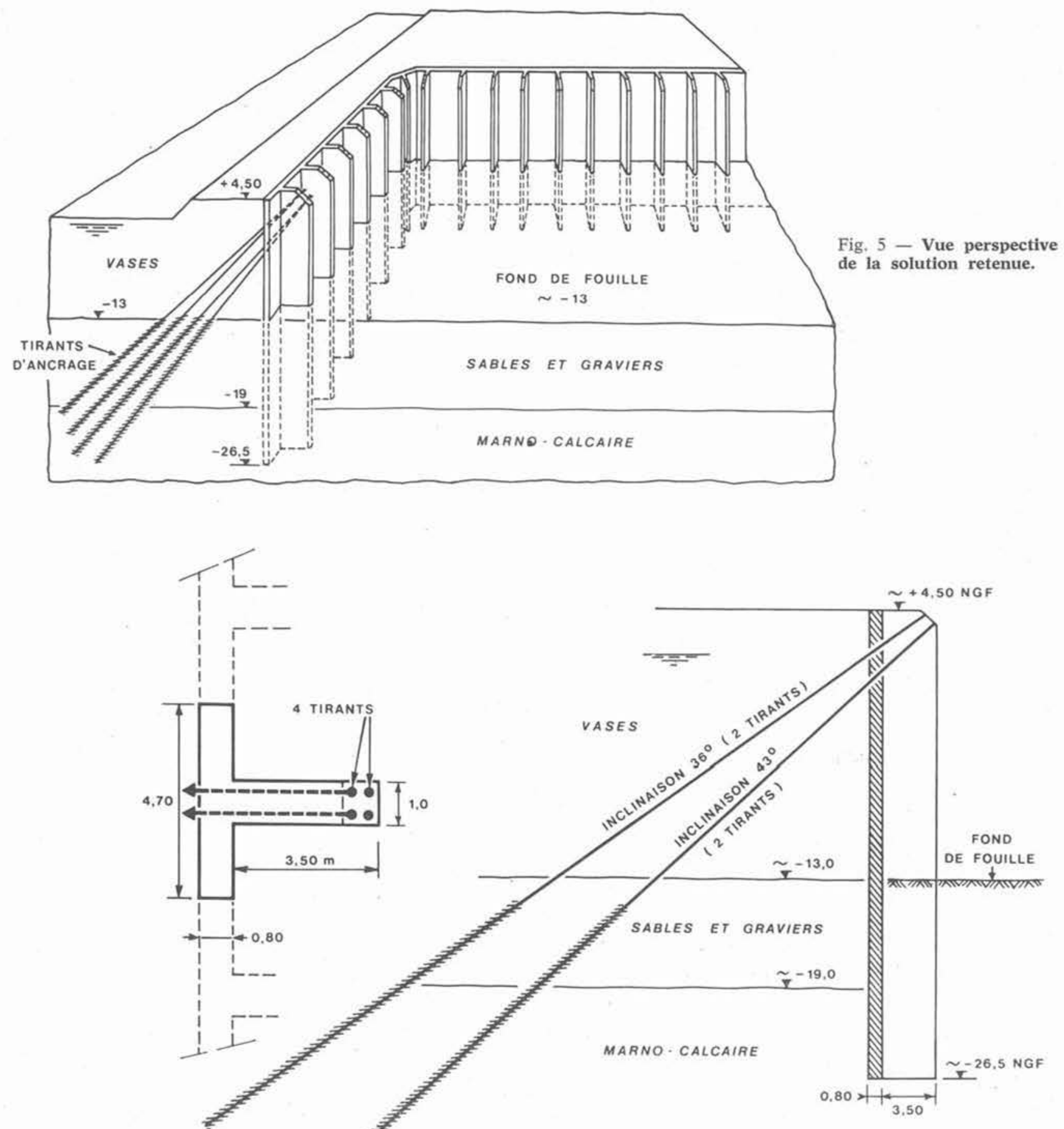

Fig. 6. - Coupe type et détail d'un contrefort.

L'ensemble du dispositif (fig. 3) est complété par vingt-quatre puits filtrants, situés à l'intérieur de la paroi et profonds de $31.5 \mathrm{~m}$. Utiles pour évacuer le débit de fuite de l'enceinte, ces puits sont essentiels pour la stabilité du fond. En effet, si le marnocalcaire est peu perméable en grand, son hétéorogénéité structurelle rend imaginable une succession de couches minces très étanches séparées par des lentilles sableuses très perméables. En supposant qu'une couche étanche se trouve située au voisinage du toit du marnocalcaire (vers la cote -20 N.G.F. par exemple), elle aurait à supporter toute la sous-pression (15 à $\left.16 \mathrm{t} / \mathrm{m}^{2}\right)$. N'ayant, au-dessus d'elle, que 6 à $7 \mathrm{~m}$ de terres $\left(4\right.$ à $\left.5 \mathrm{t} / \mathrm{m}^{2}\right)$, elle ne serait pas stable et risquerait de claquer. Sans grande conséquence sur le plan des débits d'exhaure, un tel incident serait par contre catastrophique car il décomprimerait les couches de fondation.

Ainsi, le rôle des puits profonds est d'assurer une répartition régulière des écoulements et des pressions sur l'épaisseur des marnocalcaires. 


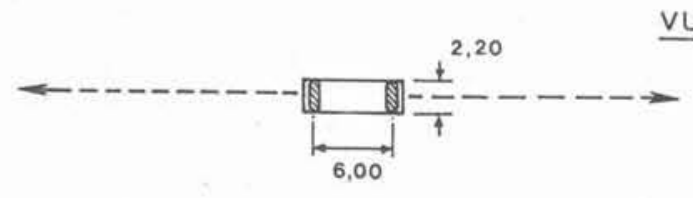

VUE EN PLAN
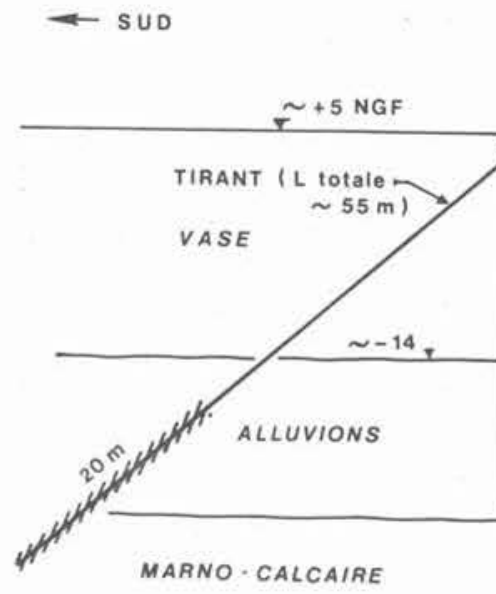

TIRANTS D'ESSAI
Fig. 7. - Vue d'ensemble du dispositif d'essai.
Fig. 8. - Détail du chevêtre et du bâti de référence.

\section{- PRINCIPE}

Les tirants, inclinés à $40^{\circ}$ en moyenne sur 1'horizontale, doivent traverser de fortes épaisseurs de vases pour atteindre les horizons d'ancrage potentiels. Ceux-ci sont constitués par les graves alluvionnaires et par le marnocalcaire sous-jacent très diversifié.

Un premier objet des essais est la définition de la résistance d'ancrage mobilisable dans ces terrains et, corrélativement, la détermination de la longueur de scellement nécessaire.

Un second objet est l'appréciation de la tenue des formations superficielles (remblais) et des vases pour définir le programme des mises en tension des tirants de l'enceinte. L'essai correspondant consiste à solliciter horizontalement une barrette moulée.

\section{- DISPOSITIF D'ESSAI}

Le dispositif d'essai est délibérément implanté dans une des zones les plus défavorables mises en évidence par les reconnaissances préalables à l'est de la future enceinte.

Il comprend (fig. 7) :

- deux barrettes $2.20 \times 0.80 \mathrm{~m}$, hautes de $30 \mathrm{~m}$, parallèles, à $6 \mathrm{~m}$ l'une de l'autre ;

- un chevêtre en béton armé $(7.80 \times 2.20 \times 1 \mathrm{~m})$ couronnant les barrettes; pour la deuxième partie des essais, ce chevêtre est conçu pour être ultérieurement coupé en deux, transversalement ;

- deux tirants symétriques, inclinés à $40^{\circ}$, ne différant l'un de l'autre que par la longueur du scellement : 20 ou $25 \mathrm{~m}$;

- un dispositif de mesure, permettant de mesurer les allongements des tirants et les mouvements tant horizontaux que verticaux du chevêtre (fig. 8), ainsi que les efforts dans les tirants.

Les tirants, du type I.R.P. 12 T 15, ont les caractéristiques suivantes :

Section d'acier : $1680 \mathrm{~mm}^{2}$

Type d'acier $\quad: 153 / 175 \mathrm{~kg} / \mathrm{mm}^{2}$
Coupe $A-A^{\prime}$
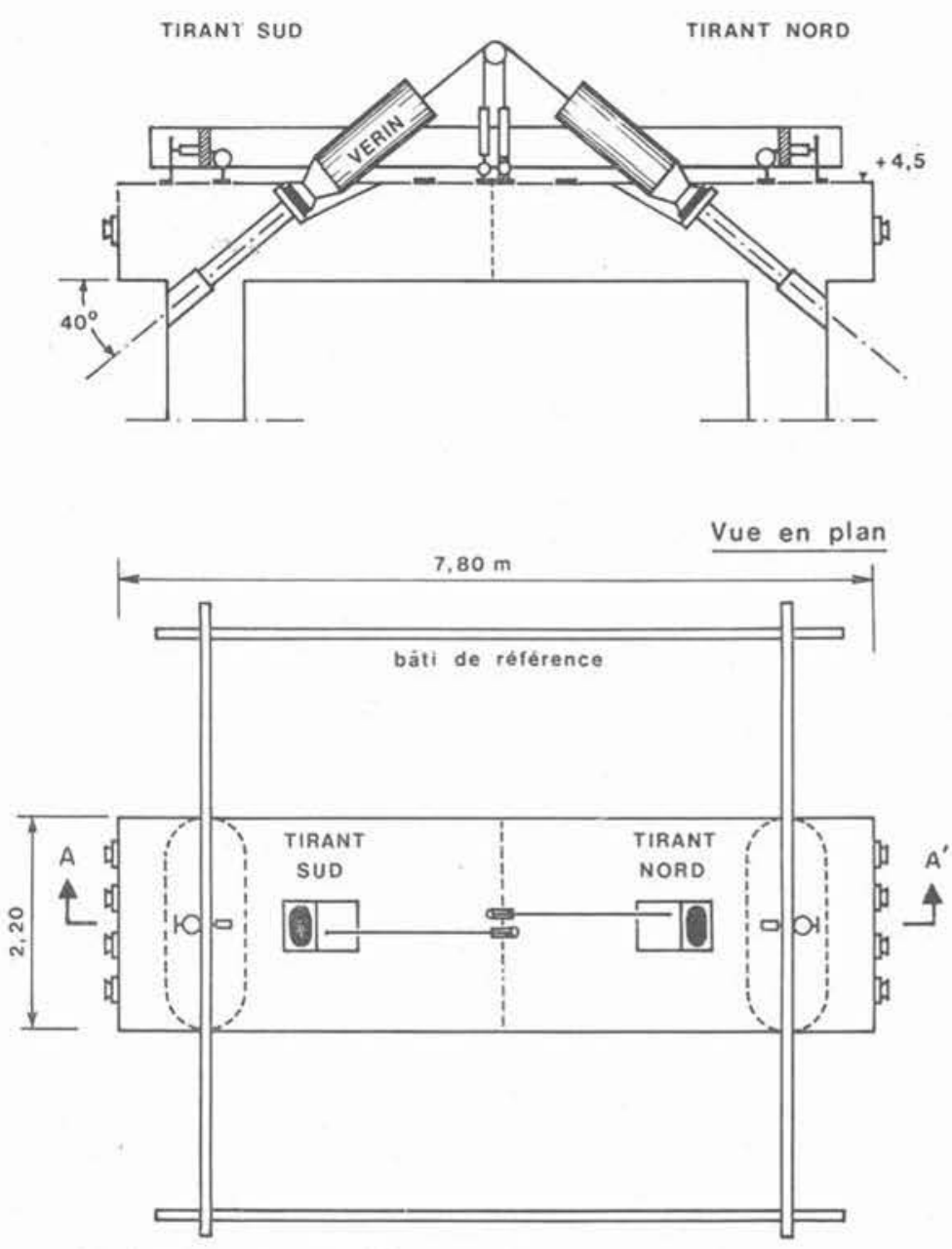

ESSAIS DE TIRANTS 


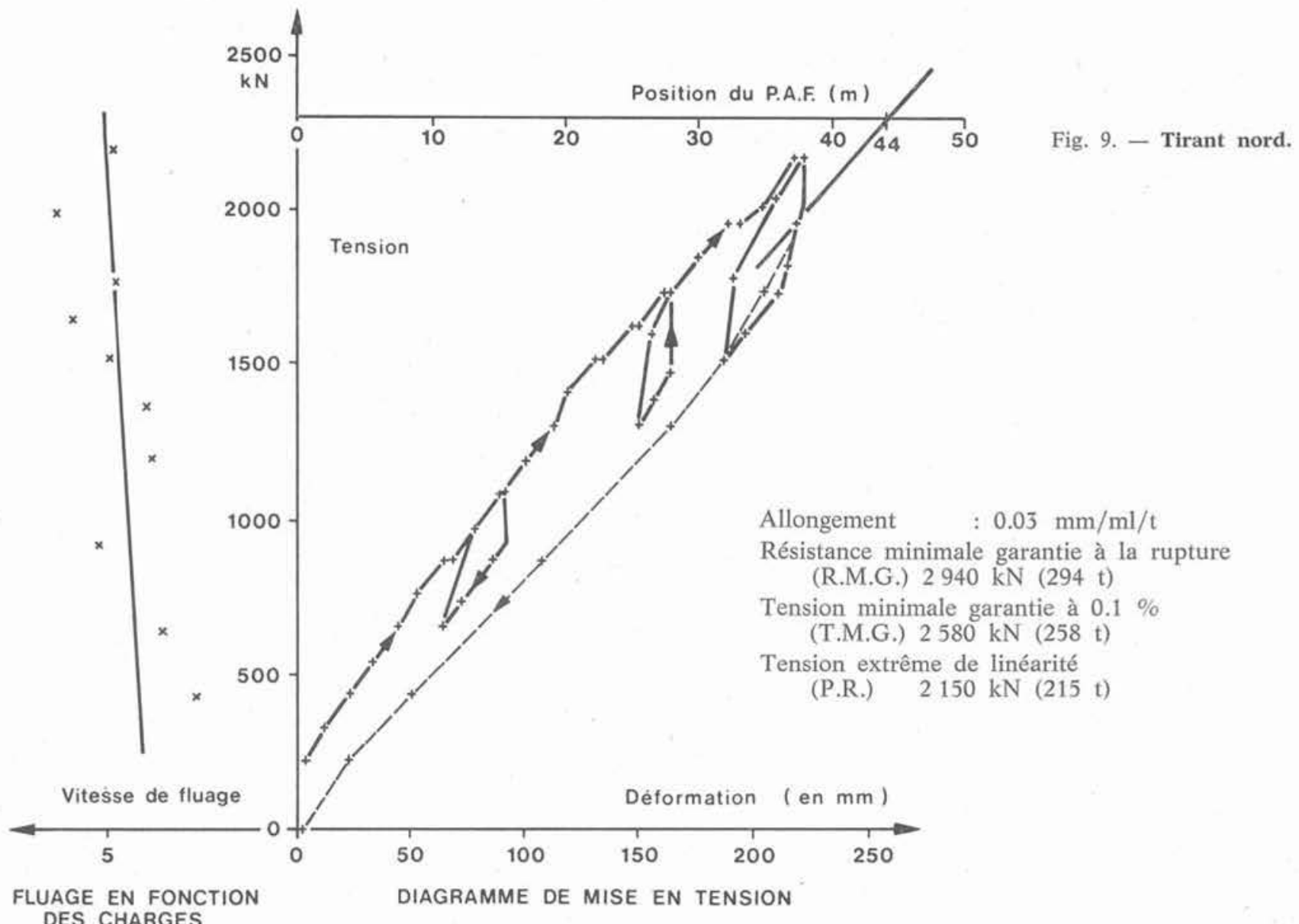

DES CHARGES
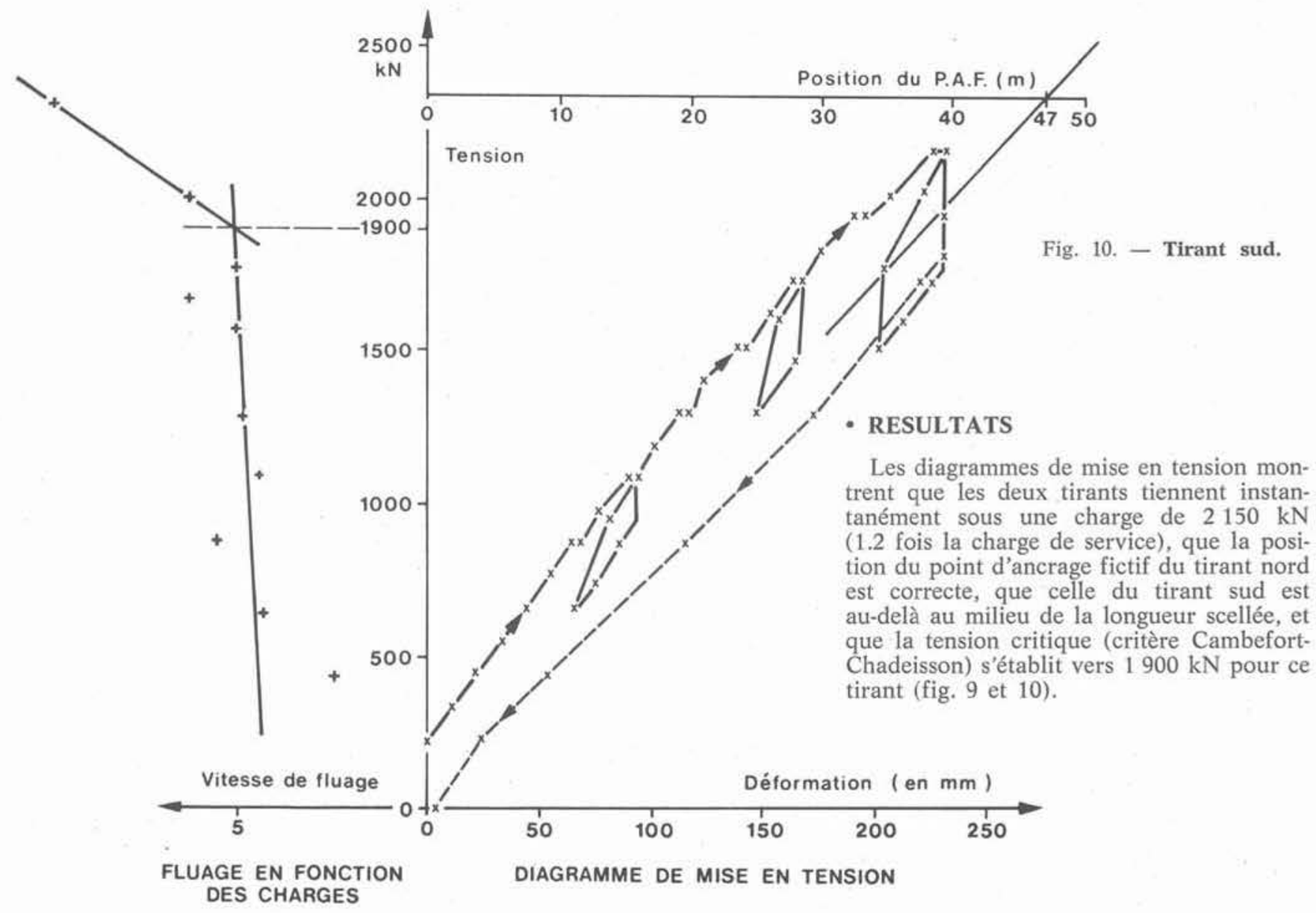
L'essai de sollicitation horizontale (fig. 11) montre que l'on doit attendre des mouvements importants dès que la vase est sollicitée en butée au-delà de $500 \mathrm{kN}$ environ par mètre linéaire de paroi.

\section{- EXPLOITATION}

En premier lieu, on décide d'adopter, pour tous les tirants, une longueur de scellement de $25 \mathrm{~m}$.

En second lieu, on constate que si on précontraint les tirants à leur valeur de service $(1930 \mathrm{kN})$, et sollicite ainsi en butée les vases à hauteur de $1250 \mathrm{kN}$ par mètre linéaire de paroi pendant toute la durée de l'exécution, on va engendrer un mouvement notable de la paroi vers la terre, suivi, lors du dragage et de la vidange, d'un mouvement en sens inverse. Cet « aller-retour " n'est pas très satisfaisant pour l'esprit : pertes de tension des tirants puis resollicitations, mouvements différentiels des plots avec cisaillement des joints, n'ont rien d'usuels.

Aussi, on choisit de limiter la tension de blocage à $50 \%$ de la tension de service (c'est-à-dire $1000 \mathrm{kN}$ par tirant).

Fig. 11. - Essai de sollicitation horizontale barrette nord.

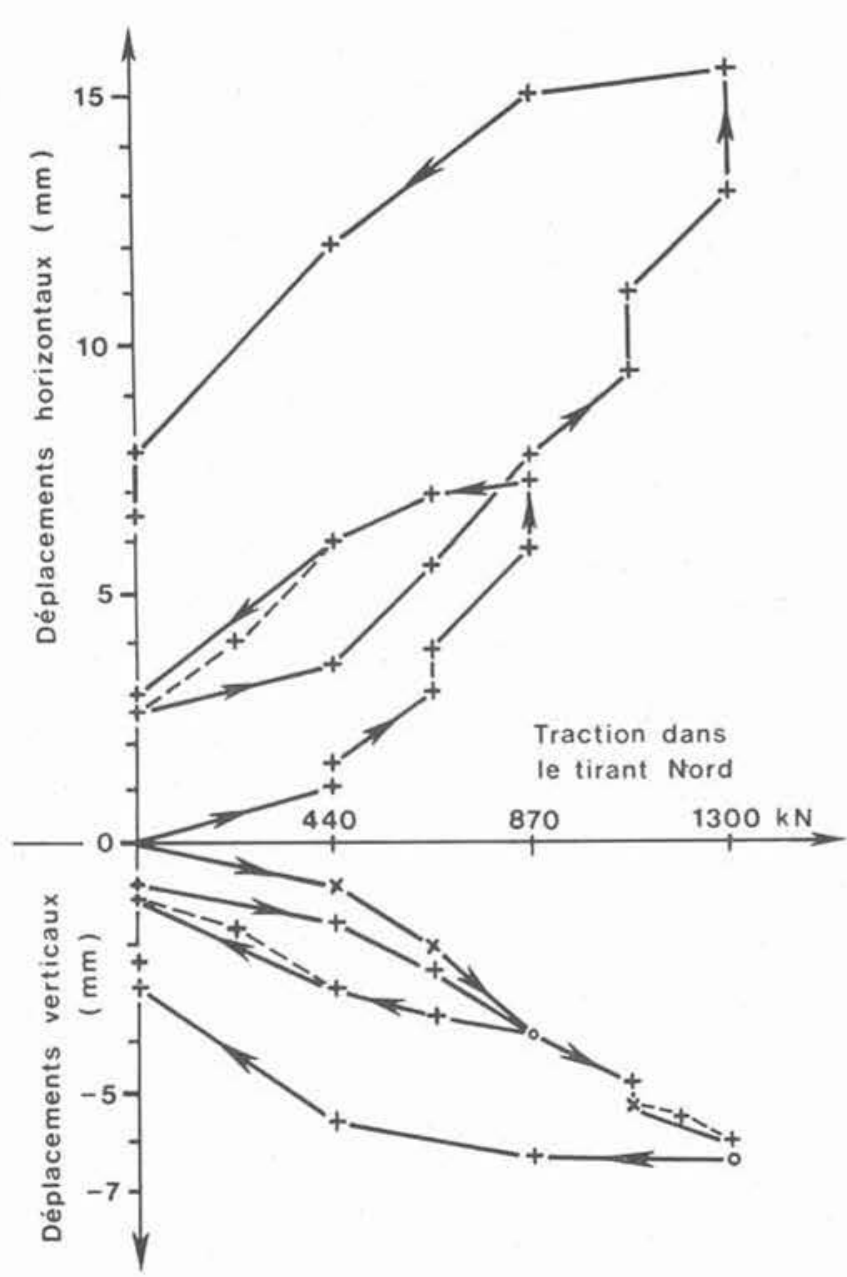

\section{MISE EN TENSION DES TIRANTS}

Les tirants sont essayés à 1.2 fois la tension de service en appliquant la méthode du cycle (Rceommandations TA 77).

Des processus particuliers sont mis au point afin d'éviter au maximum des différences de sollicitation importantes entre plots voisins. A cette fin, on teste les tirants par paire, à cheval sur deux plots, en n'en sollicitant qu'un à la fois sur chaque plot.

Il importe que les quatre tirants d'un même plot aient sensiblement la même tension, $50 \%$ de la charge de service, mesurée après le blocage de tous. Des essais sont conduits pour mesurer la perte de tension engendrée dans un tirant donné par la mise en service d'un autre du même plot. Cette perte est de l'ordre de 5 à $6 \%$ de la charge de service. Ceci conduit aux valeurs successives de blocage suivantes : $60 \%$ sur le tirant A, $55 \%$ sur le tirant C, $52 \%$ sur le tirant B, enfin $50 \%$ sur le tirant D.

On doit ajouter enfin les essais usuels faits systématiquement tous les $n$ tirants : étude fine du fluage et de la charge critique, essai spécial à $8000 \mathrm{kN}$ sur quelques plots (pendant quelques minutes).

\section{DISPOSITIF DE CONTROLE}

En dehors de tous les contrôles qu'il est d'usage de faire en cours de chantier, et qui constituent les règles de l'Art, un dispositif est mis en œuvre pour suivre paroi, tirants et rabattements pendant les phases cruciales du dragage, de la vidange et du terrassement à $\sec$ (fig. 12) :

- des potences métalliques (23) pour les déplacements horizontaux et les rotations en tête de la paroi ;

- des tubes verticaux (14) aux nœuds des contreforts pour mesures clinométriques ;

- des cales dynamométriques (Dynasolf et Glötzl) (33) pour les tensions dans les tirants;
- un ensemble de points de mesure des niveaux piézométriques et des débits d'exhaure.

Ce dispositif trouve ses justifications en considérant d'une part, les dimensions exceptionnelles du soutènement, d'autre part, le processus particulier de mise en tension des tirants. On s'attend lors du terrassement à noter des déplacements de la paroi vers la fouille, d'une amplitude voisine en tête et en pied, de l'ordre de 6 à $8 \mathrm{~cm}$ selon la valeur des sollicitations réelles. Un tel mouvement, compatible avec l'environnement, doit permettre réellement l'obtention de la poussée minimale. 
NORD

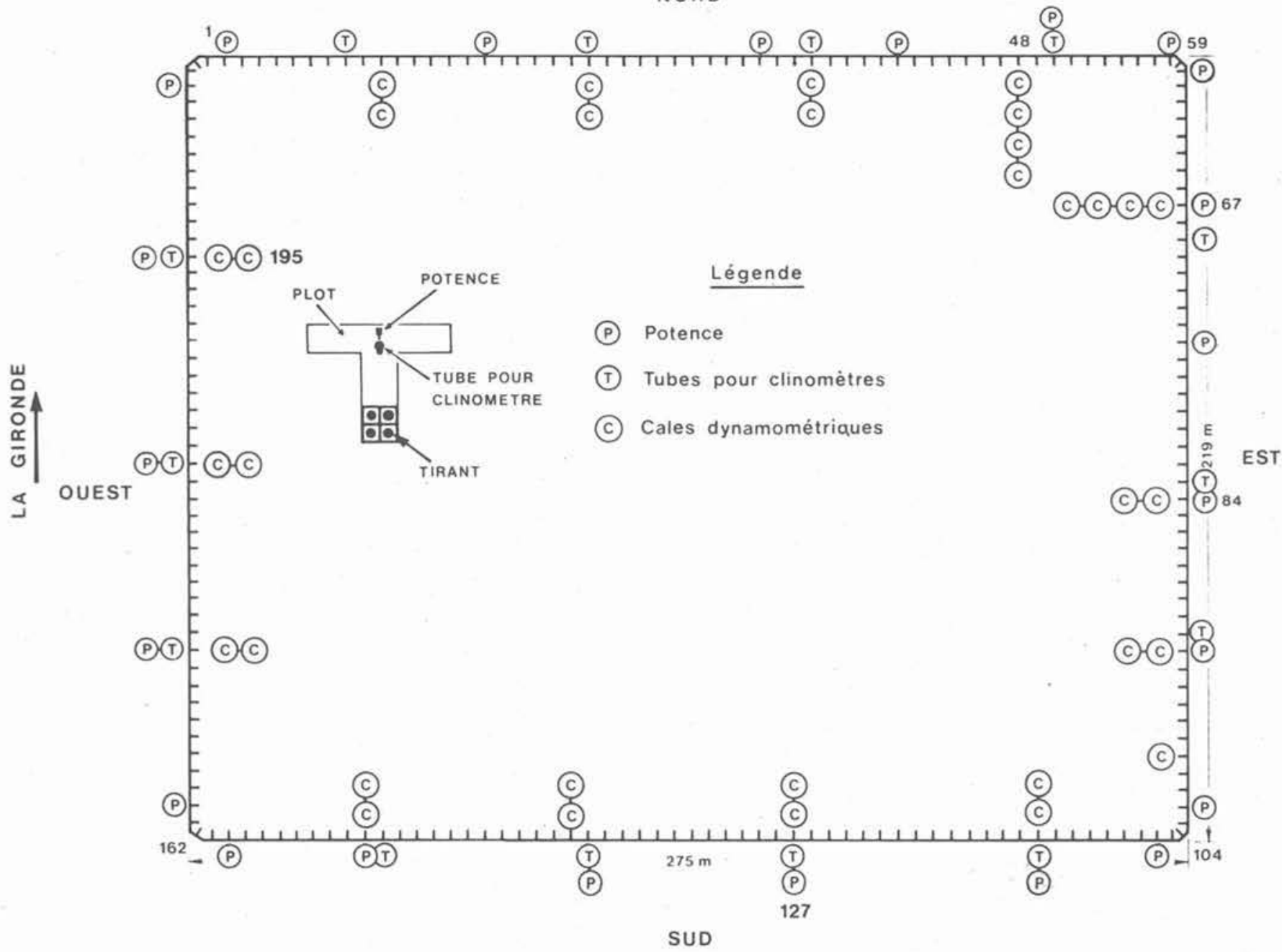

Fig. 12. - Dispositifs de mesure sur tirants et parois

\section{RESULTATS}

Les figures 13 et 14 représentent, en fonction de l'avancement des travaux, les mouvements de la paroi et les tensions dans les tirants dans les plots 127 (paroi sud) et 195 (paroi ouest), choisis comme caractéristiques des observations faites sur vingt-trois profils.

On remarque :

- la difficulté que l'on éprouve, sur un chantier, à conduire des mesures dès l'origine des travaux.... on installe le dispositif, telle ou telle mancuvre le met hors de service, on recommence... et on perd les petits mouvements d'origine ( $1 \mathrm{~cm}$ peut-être) ;

- le délai de réflexion de la paroi, qui amorce ses mouvements plusieurs jours après être sollicitée ;

- l'influence du dernier mètre terrassé (à sec), qui représente à lui seul une augmentation considérable des efforts et des mouvements (plus de $10 \%$ );

- la concordance entre les phénomènes attendus et la réalité observée.
Le figure 15 représente les mouvements maximum de la paroi. Sa forme en " as de carreau " est conforme à la structure : les quatre angles sont des points fixes. La paroi ouest (côté Gironde) a été plus sollicitée que les autres : terrassement à une cote plus basse ( $-13.50 \mathrm{NGF}$ ), niveau d'eau de la nappe de surface plus élevé $(+2.50 \mathrm{NGF})$. Le plot 50 est un cas particulier : un défaut d'étanchéité au droit d'un joint a été traité par injection derrière la paroi et le coulis injecté a poussé un peu trop sur celle-ci.

Les mesures d'inclinaison sont restées dans le domaine de l'imprécision (1/1000 environ) et n'ont pas révélé des mouvements spectaculaires.

Le système de rabattement-décompression du fond de fouille fonctionne correctement. Les débits mesurés restent nettement inférieurs à $200 \mathrm{~m}^{3} / \mathrm{h}$, débit attendu comme normal correspondant à $50 \%$ de la capacité de pompage installé. Les niveaux piézométriques - en particulier un point de mesure au centre de la fouille - sont également très satisfaisants.

\section{COMMENTAIRES}

1) Il est, à première vue, assez étonnant que la vidange de la fouille sur $12.50 \mathrm{~m}$ n'ait entraîné qu'un si faible accroissement de tension des tirants (moins de 10 tonnes) accompagné d'un faible avancement de la paroi (de l'ordre de 8 à $10 \mathrm{~mm}$ ). 
\begin{tabular}{|l|l|l|l|l|l|l|l|l|l|}
\hline AVRIL & MAI & JUIN & JUIL. & AOÛT & SEPT. & OCT. & NOV. & DEC. & JANV. \\
\hline
\end{tabular}
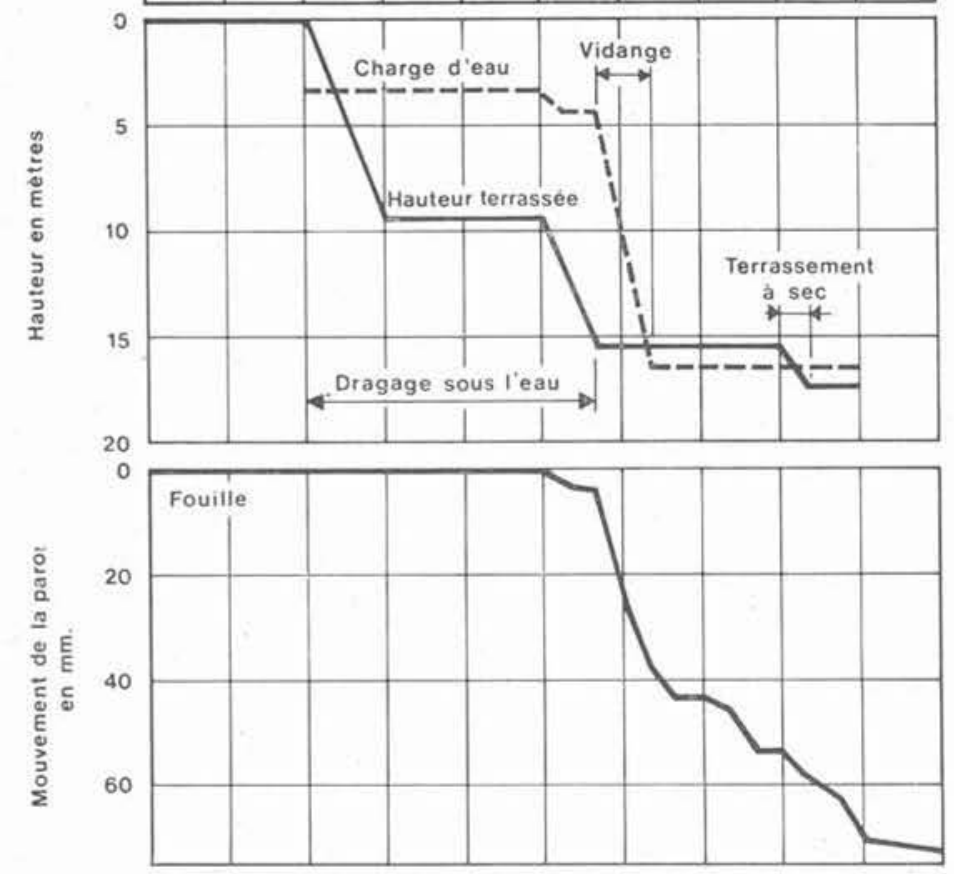

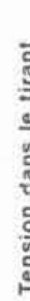

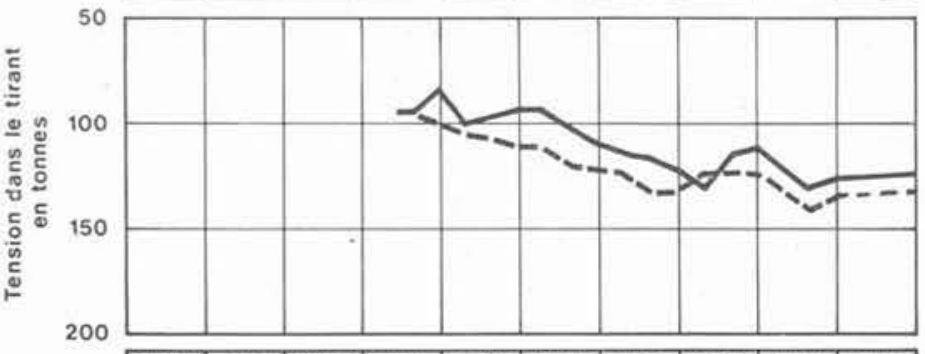

\begin{tabular}{|l|l|l|l|l|l|l|l|l|l|}
\hline AVRIL & MAI & JUIN & JUIL. & AOÜT & SEPT. & OCT. & NOV. & DEC. & JANV. \\
\hline
\end{tabular}
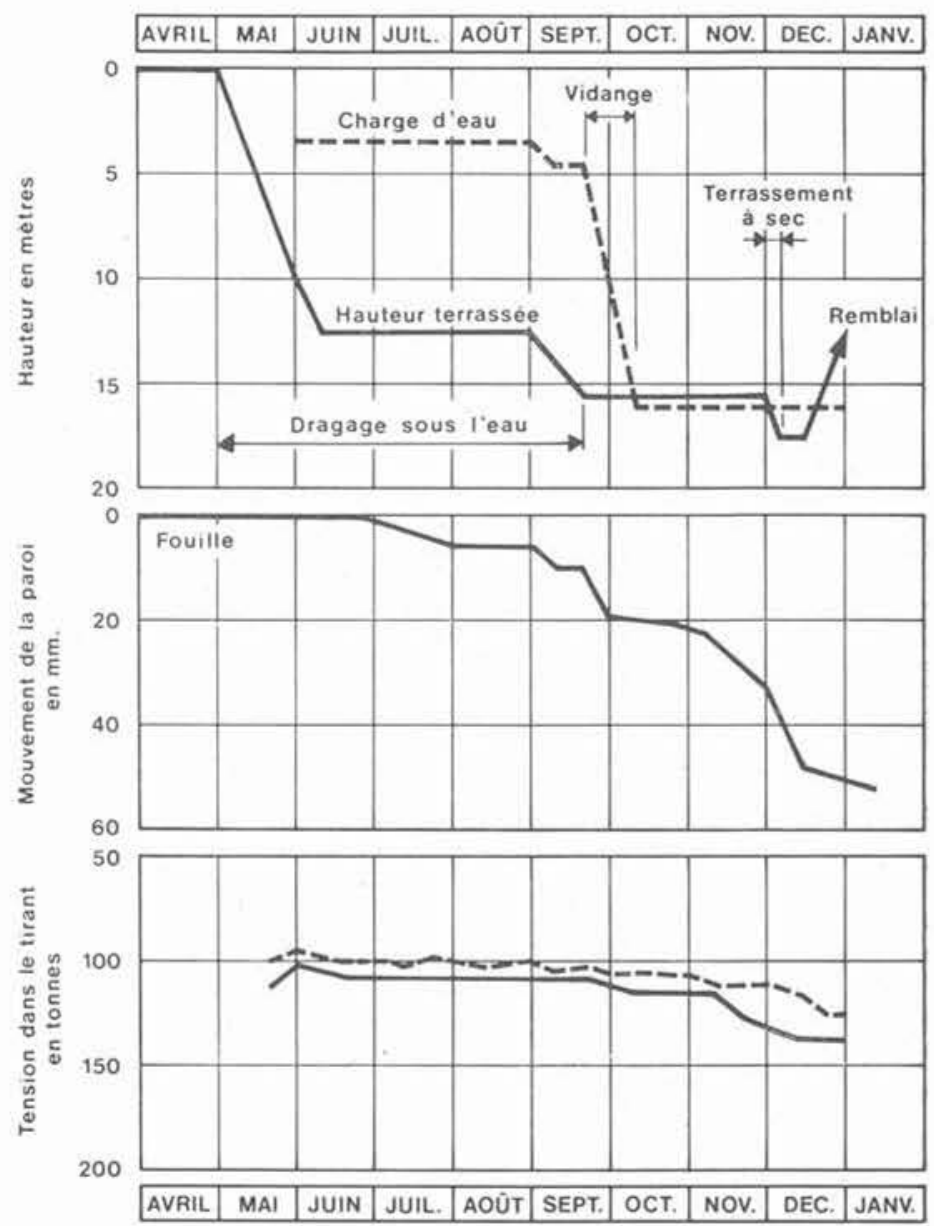

Fig. 14. - Mouvements paroi et tension tirants. Plot 127.

Fig. 13. - Mouvements paroi et tension tirants. Plot 195.

NORD

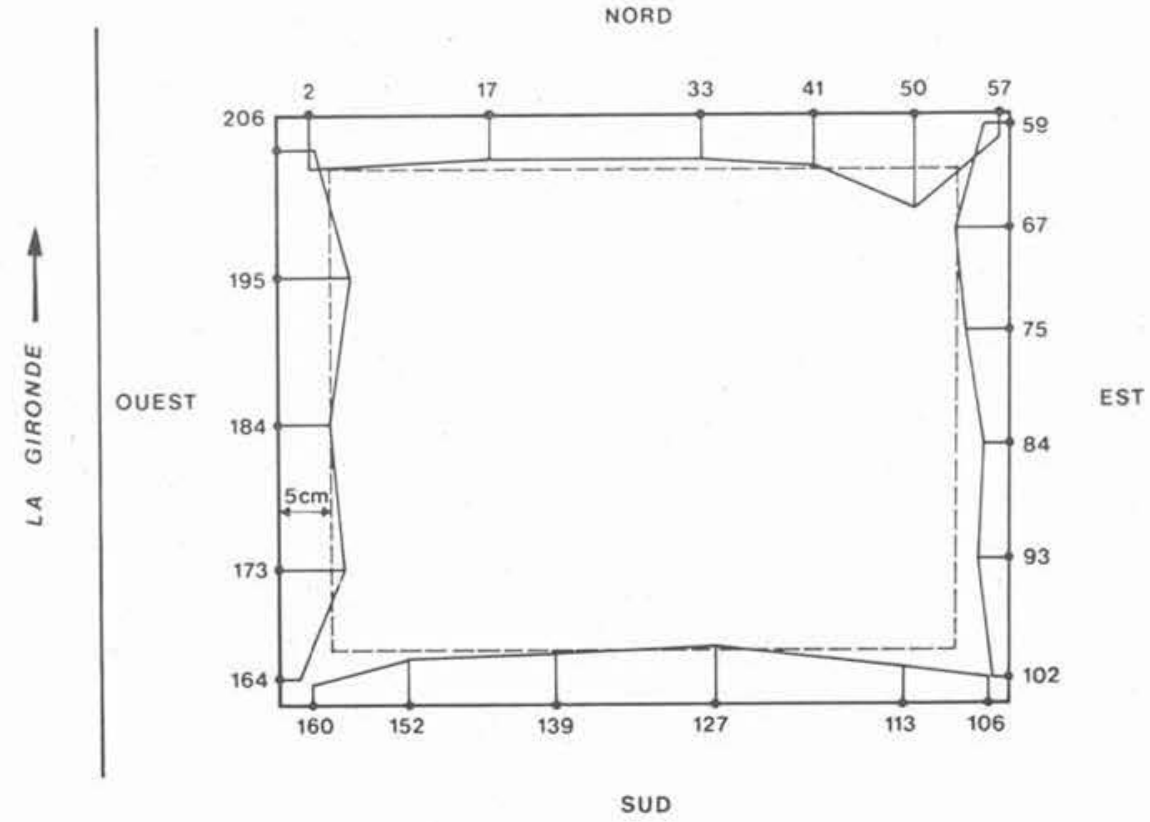

Fig 15. - Mouvements de la paroi. 
L'explication qui paraît la plus simple est de considérer que la paroi est quasiment un solide indéformable (la flexion propre de la paroi correspond à des flèches maximum de l'ordre de $1 \mathrm{~cm}$, qu'on peut négliger ici). Ce solide indéformable est appliqué sur le sol par la mise en traction des tirants, côté extérieur de la fouille (phase 2) (fig. 4).

En fin de phase 1, le coefficient de poussée vaut environ 0,5 sur chaque face de la paroi. En fin de phase 2 , ce coefficient a évolué d'une part à l'extérieur (butée sans déformation) vers $\mathrm{K}_{e}=1$, et d'autre part à l'intérieur (poussée minimale) vers $\mathrm{K}_{i}=0.25$

Quand on vidange la fouille (phase 3), on met en mouvement la paroi vers l'intérieur, $\mathrm{K}_{e}$ glisse vers 0.25 valeur de la poussée : le $\Delta \mathrm{K}_{e}$ balance à peu près la pression de l'eau qui vient d'être vidangée, d'où une faible augmentation de la force du tirant.

Cette analyse conduit à penser que l'on passe d'un équilibre de butée à l'équilibre de poussée au prix d'un très faible mouvement.
2) Le mouvement de la tête de la paroi et l'accroissement de la force dans le tirant sont en relation théoriquement linéaire.

Dans $50 \%$ des profils étudiés, on vérifie aisément que la relation est conforme aux paramètres (longueur libre du tirant, caractéristiques de déformation de l'acier en fonction de sa tension).

Dans $50 \%$, on ne retrouve pas clairement cette relation, la traction dans le tirant restant en deçà. L'explication de ce phénomène peut venir soit d'un fluage au niveau des têtes, soit d'un mouvement vertical descendant de la paroi. Nous ne possédons pas de mesures de cet éventuel mouvement, qui n'est pas impossible compte tenu des efforts engendrés.

3) En conclusion, nous souhaitons mettre en lumière l'augmentation de sécurité, sur la sollicitation réelle des tirants, que le processus adopté a offert. A notre connaissance, il s'agit du premier exemple, à cette échelle, d'un tel parti de construction et nous pensons qu'il doit pouvoir s'appliquer chaque fois qu'on est en présence d'une structure de soutènement simple dont les mouvements éventuels sont compatibles avec l'environnement. 